\title{
NANOCRYSTAL SUSPENSION OF CEFIXIME TRIHYDRATE PREPARATION BY HIGH-PRESSURE HOMOGENIZATION FORMULATION DESIGN USING $2^{3}$ FACTORIAL DESIGN
}

\author{
RAJVEER BHASKAR ${ }^{* 1}$, PRAKASH HIRAMAN PATIL ${ }^{1}$ \\ 1Department of Pharmaceutics and Quality Assurance, R C Patel Institute of Pharmaceutical Education and Research, Near Karvand Naka, \\ Shirpur, District-Dhule, Maharashtra 425405 \\ Email: bhaskar007.raj@gmail.com
}

Received: 21 Apr 2017 Revised and Accepted: 13 Jul 2017

\section{ABSTRACT}

Objective: In the present study, nanocrystal suspensions of cefixime trihydrate were prepared with the objective of providing increased solubility and stability with their nanoscopic size and thus developing the formulation of enhanced bioavailability potential.

Methods: Nanocrystal suspensions were prepared by high-pressure homogenization technique using PVP K-30 as a stabilizer and evaluated for particle size, polydispersity index, zeta potential, permeation and drug release.

Results: Particles of average size $143.5 \mathrm{~nm}$ having a polydispersity index of 0.269 were produced. Zeta potential was found to be $-36.6 \mathrm{mV}$ and the formulation was found stable on the basis of results obtained from differential scanning calorimetry and Fourier transform infrared spectroscopy studies. Optimized formulation showed $89.79 \%$ and $88.38 \%$ drug lease and permeation respectively.

Conclusion: The drug release and ex-vivo permeation studies revealed enhanced permeation of drug, as desired, indicating its potential for an attempt towards successful nano crystal formulation.

Keywords: Nanocrystal suspension, Cefixime trihydrate, High-pressure homogenization, Drug release

(C) 2017 The Authors. Published by Innovare Academic Sciences Pvt Ltd. This is an open access article under the CC BY license (http://creativecommons.org/licenses/by/4.0/) DOI: http://dx.doi.org/10.22159/ijpps.2017v9i9.19319

\section{INTRODUCTION}

A major challenge in oral drug delivery is the low bioavailability of water insoluble therapeutics [1-2]. Two known mechanisms to increase the bioavailability of water insoluble drugs are to increase the surface area and to create stable amorphous forms [3-4]. A major hurdle that has prevented the commercialization of many promising poorly soluble drugs is dissolution rate limited bioavailability. Many approaches have been developed to improve solubility and to enhance the dissolution rate and oral bioavailability of poorly soluble drugs e. g. salt formation [5-7] solid dispersion [810] inclusion complex [11]. Cefixime trihydrate, an orally active third generation cephalosporin antibiotic, is extensively used for the treatment of urinary tract infection (UTI), upper and lower respiratory tract infection, acute otitis media, and Gonococcal urethritis [12]. On an oral administration, it gets slowly and incompletely absorbed from GIT. Antibacterial activity of cefixime exhibited by interfering with bacterial peptidoglycan synthesis after binding to the $\beta$-lactam binding proteins [12-13]. Cefixime fall in a low solubility and low permeability antibiotic drug. Bioavailability of a drug is restricted due to low solubility and due low solubility of cefixime, its bioavailability is only $30-40 \%$ if given orally and show maximum peak plasma concentration within 2-4 h [14-15]. Here, it is necessary to realize that an ionic charge on cefixime creates difficulty to cross mucosal membrane of the intestine, resulting in poor bioavailability of 40-50\% [16-17].

The poor water solubility of drugs such as CFX is a major hurdle for drug formulation. It results in poor bioavailability and therapeutic failure of the drug due to their inability to adequately reach their target sites [18-19]. Nanocrystal suspensions (NCS) are promising candidates that can be used to enhance the dissolution rates of such poorly soluble drugs [20-22]. In NCS, the drug is maintained in the required crystalline state with reduced particle size which results in an increase in surface area and the creation of high energy surfaces due to the disruption of drug micro crystals to nanoscopic particles (NPs). In addition, particles size reduction to the nanoscale decreases the diffusional distance on the surface of drug NPs, thus enhancing the concentration gradient. Moreover, NPs tend to have an increased adhesiveness to surfaces, cell membranes and intestinal wall, which contributes to increased drug absorption and reduced inter-individual variation with overall enhanced drug bioavailability [23]. Despite recent progress and numerous researches on NCS technology, stability issues limit their applications in pharma industries [24-25]. Aggregation of the nanoscopic particles is the main stability issue which can occur during preparation process or storage and it is due to the Ostwald ripening phenomenon [26]. Among various ways to overcome NCS stability problem such as stabilizer addition [26-28], solidifying is one of the main solutions $[24,27,29]$. The solid state is preferred over the aqueous nanosuspensions, due to the fact that aggregation and other instability factors are significantly decreased. Therefore, nano suspensions are commonly converted to the solid state which can be further formulated into another dosage form [30-31]. In the present study, an attempt was made to formulate cefixime trihydrate in nanocrystal suspension form in order to overcome the solubility and formulation related issue and same was supported by obtained results.

\section{MATERIALS AND METHODS}

Cefixime trihydrate was purchased from Parabolic Drug Ltd., Mumbai, India. PVP K-30, SLS, Tween 80 were purchased from Moylchem, Mumbai, India. Dialysis membrane and polyvinyl alcohol (PVA), was procured from Hi-media Labs New Delhi, India. All analytical grade chemicals and reagents were used in the present study.

\section{Selection of stabilizer}

Nanocrystal suspensions were prepared using different stabilizers alone and in combination and were evaluated with respect to the particle size, PDI using Malvern Zetasizer Nano-ZS90, UK. Selection of the stabilizer was made based on minimum particle size and PDI.

\section{Preparation of cefixime trihydrate nano crystal suspensions}

Nanocrystal suspensions were prepared by adding the cefixime trihydrate in water containing stabilizer which was added for the stabilization of the resultant suspension. This suspension of 
insoluble drug was first homogenized by using ultra-turrex T-25 (IKA, Germany) at 10,000 rpm for 15 min then passed through a high-pressure homogenizer $(\mathrm{HPH})$ at predetermined optimized pressure and cycles (temperature maintained below 10 a during ultra-turrex and HPH). On the basis of literature survey and trail batches, various critical process variables and formulation variables were optimized by using $3^{2}$ factorial design with Design Expert 10.0.6 software (Stat-Ease, Inc., USA). The pressures, number of cycles in HPH and stabilizer concentration were selected as undependable variables and response on particle size, PDI and drug release were investigated.

\section{Particle size, polydispersity index and zeta potential}

The mean particle size analysis (Z-average) and polydispersity index (PDI) were determined by dynamic light scattering principle (Zetasizer Nano ZS90, Malvern Instruments UK). The samples were diluted as per user manual and results obtained for size and PDI were recorded. The test was conducted at an angle of $173^{\circ}$ backscatters (NBS default) with measurement position set at 4.65 $\mathrm{mm}$. Measurements for zeta potentials were carried out at 25 ? using double distilled water as a dispersant in a clear disposable zeta cell.

\section{Differential scanning calorimetry (DSC)}

A differential scanning calorimeter (Mettler Toledo DSC 1) with STAReSW 10 software was used for recording and processing DSC thermograms of cefixime, PVP K-30and optimized NCS. The accurately weighed sample (3-5 mg) was placed in an aluminium pan. The experiment was carried out in a nitrogen atmosphere at a scanning rate of $10^{\circ} \mathrm{C} / \mathrm{min}$ in the range of $40-340^{\circ} \mathrm{C}$.

\section{Fourier transform infrared spectroscopy (FTIR)}

Fourier transform infrared spectra of pure drug, a physical mixture of drug and selected stabilizer and optimized formulation were recorded using FTIR spectrophotometer (FTIR-8400S, Shimadzu, Japan) in the range of 4000-400 $\mathrm{cm}^{-1}$ and compared for any significant change.

\section{Scanning electron microscopy (SEM)}

The morphology of the cefixime trihydrate and optimized formulation were investigated by scanning electron microscopy (SEM, JOEL JSM-5600) at an accelerating voltage of $1.0 \mathrm{kV}$. Double coated carbon conductive tabs are mounted on SEM sample stubs.

\section{Saturation solubility of CEF and CEF NCS}

The saturation solubility of the pure drug in water was determined by the traditional shake flask method. According to this method excess amount of pure drug and optimized formulation were added in $10 \mathrm{ml}$ deionized water in a capped vial, which was then placed in a temperature-controlled shaking water bath at $37^{\circ} \mathrm{C}$ and $100 \mathrm{rpm}$ for $24 \mathrm{~h}$. The saturation is confirmed by the presence of undissolved material. After separation of the solid by filtration, the concentration of the drug in the filtrate was determined by UV-Visible spectrophotometer (Shimadzu 2420, Japan) at $288 \mathrm{~nm}$.

\section{Drug release study}

In vitro drug diffusion profile was obtained by dialysis-bag/dialysissac method [32]. Optimized NCS and raw drug suspension were filled in activated dialysis membrane bags (dialysis membrane 110 (LA 395), Hi-Media) and suspended in glass beakers containing methanolic phosphate buffer saline (PBS) ( $\mathrm{pH}$ 6.8). Cefixime trihydrate has limited solubility in the buffer but is soluble in methanol; hence methanol was added to PBS pH 6.8 to maintain the perfect sink conditions [33].

The beakers were placed on magnetic stirrers and stirred with magnetic beads and were covered with paraffin film to prevent any evaporative loss during the experimental run [34]. Aliquots were withdrawn from the receptor compartments at periodic time intervals for $24 \mathrm{~h}$ and replaced with equivalent amounts of fresh diffusion medium. The aliquots were analysed spectrophotometrically at $288 \mathrm{~nm}$. All the experiments were performed in triplicate.

\section{Permeation study}

Optimized NCS formulation permeation study was conducted on wistar albino rats against drug solution as a control for permeation across rat intestine. The rats kept on overnight fasting were sacrificed and their intestine was excised into $5 \mathrm{~cm}$ long pieces from distal to the duodenojejunal flexure and washed thoroughly with saline solutions. Each piece of intestine was legated at one end with silk thread, filled with NCS (equivalent to $10 \mathrm{mg}$ of drug) and the other end was legated to form a balloon-shaped sac. The sac containing NCS were immersed in Krebs solution (100 ml) contained beaker with stirring (200 rpm). Aliquots $(2 \mathrm{ml})$ were withdrawn from beaker at a specified time interval, i.e. 15, 30, 45, 60, 90 and $120 \mathrm{~min}$ and the same volume of fresh buffer were added to a beaker in order to maintain sink condition.

\section{RESULTS AND DISCUSSION}

\section{Selection of stabilizer}

For the selection of stabilizer, NCS were prepared with six different stabilizers and were evaluated for particle size and PDI. Minimum particle size and PDI was achieved with PVP K-30. Stabilizers are used to provide short term and long-term storage stability for nanosuspension along with the stabilization of nanocrystals during particle production. Non-ionic stabilizers are reported to sterically stabilize the nanoparticles [35]. In general, bulky and non-ionic surfactants are reported to be less toxic than single-chain and ionic surfactants [36-38]. Hence, PVP K-30 was selected for further investigations for the formulation.

\section{Optimization of process and formulation variables}

Critical process and formulation variables which may have a significant effect on the critical quality attributes were identified for each step involved in the formulation, that is, stabilizer concentration, pressure and number of cycles in HPH. Preliminary optimization of stirring time and RPM of high-speed homogenizer (IKA, Germany) was carried out by conducting the experiments at three different RPM (5000 to 15000) for three different time durations ( 10 to $20 \mathrm{~min}$ ) at room temperature. From the results obtained (results not shown) in terms of particle size and PDI, it was observed that as the homogenization time and/or homogenization speed increased, the particle size decreased which may be attributed to increased force of deformation at higher speed leading to smaller particles. It was also observed that with the increase in homogenization time PDI also increased which may be due to the formation of foam and more aggregation in the formulation. Best results were obtained by stirring at $10000 \mathrm{RPM}$ for $15 \mathrm{~min}$.

Hence further investigations were done with homogenization/ stirring speed of $10000 \mathrm{RPM}$ for $15 \mathrm{~min}$. Critical process variables involved during the HPH were pressure, a number of cycles and stabilizer's concentration. These were optimized using 23 factorial design with Design Expert 10.0.6 software (Stat-Ease, Inc., USA). Eight runs were carried out with pressure ( 400 to 800 bars) number of cycles (1 to 5 ) and stabilizer concentration ( 30 to $60 \%$ wt of drug) as independent variables and particle size dependent variables. The full factorial design used for optimization of process variables is shown in table 1 . ANOVA was applied to determine the significance and the magnitude of the effects of the variables and their interactions showed in table 2 . Full model equation for particle size in terms of coded factors was obtained as.

$$
\text { Sqrt (Particle size) }=+17.96-0.82 * A-1.14 * B-2.32 * \text { C. (1) }
$$

The individual effect of surfactant concentration (A) was found to be insignificant ( $\mathrm{p}$ value $=0.1372$ ), a number of cycles $(B)$ was found to be marginally significant ( $p$ value $=0.0618$ ) and of pressure $(C)$ was significant $(p$ value $=0.0064)$. Pareto chart reveals that pressure has most significant effect on particle size compared to HPH cycle and stabilizer concentration as given in fig. 1 . The regression model obtained was used to generate the contour and 3D surface plots as shown in fig. 2 for analysing interactions of the independent factors reveals as the pressure and/or number of cycles were increased, the particle size was found to be reduced. 
Table 1: $2^{3}$ factorial design with coded values used for optimization of formulation variables (independent variable: concentration of stabilizer, cycle and pressure in $\mathrm{HPH}$, dependent variable: particle size)

\begin{tabular}{lllll}
\hline Run & Stabilizers conc. & Cycle & Pressure & Response (PS in nm) \\
\hline 1 & +1 & -1 & +1 & 291.6 \\
2 & +1 & +1 & -1 & 143.5 \\
3 & -1 & -1 & +1 & 309.3 \\
4 & -1 & +1 & -1 & 399.1 \\
5 & +1 & +1 & -1 & 376.3 \\
6 & -1 & +1 & +1 & 253.3 \\
7 & +1 & -1 & -1 & 403.7 \\
8 & -1 & -1 & -1 & 468.2 \\
\hline
\end{tabular}

Table 2: ANOVA for response surface model for response (Particle size)

\begin{tabular}{lllll}
\hline Term & & Sum of squares & \% contribution \\
\hline A- & stabilizer & 5.40 & 8.30 & P value \\
B- & Cycle & 10.40 & 15.98 & 0.1372 \\
C- & Pressure & 42.99 & 66.07 & 0.0618 \\
AB & & 0.76 & 1.16 & .0064 \\
AC & & 0.68 & 1.04 & - \\
BC & & 2.43 & 3.34 & - \\
ABC & & 2.41 & 3.71 \\
\hline
\end{tabular}

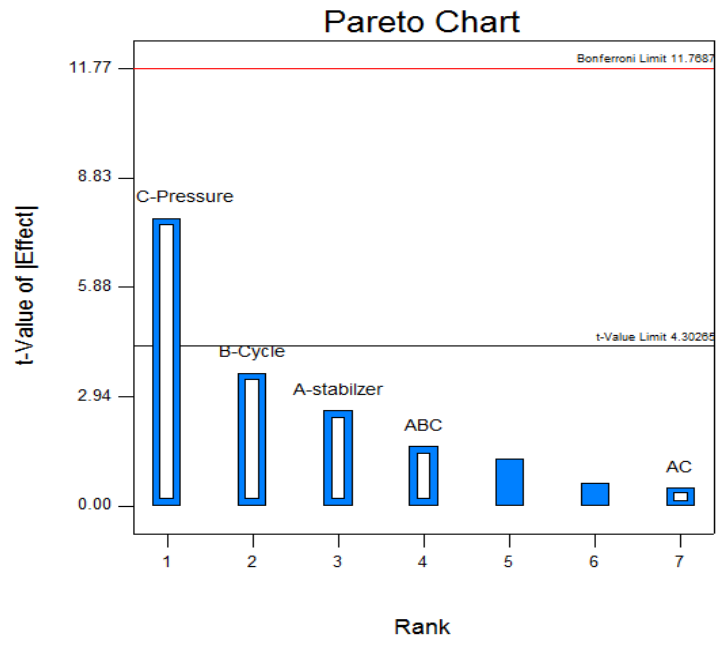

Fig. 1: Relative size of different factors on response
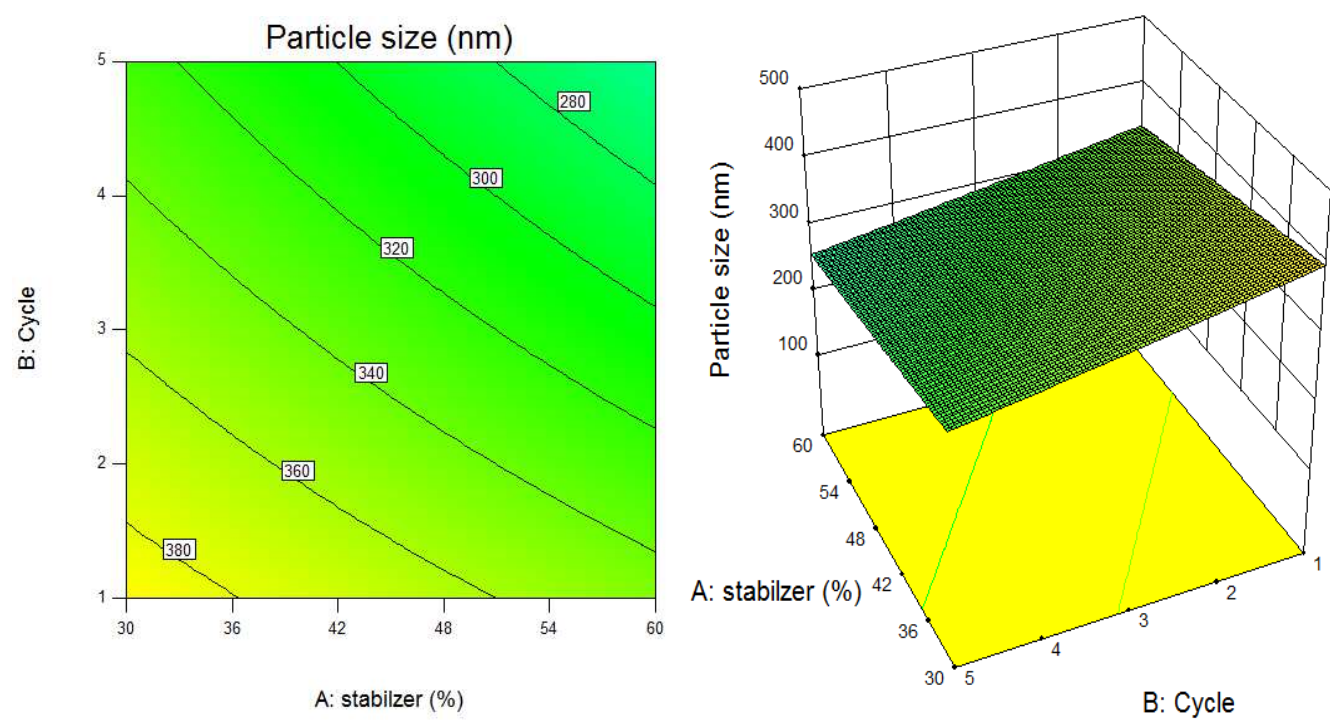

Fig. 2: Contour and 3D response surface plot showing effect of pressure and cycle of high pressure homogenization on particle size 


\section{Particle size, polydispersity index (PDI) and zeta potential}

The average particle size, PDI and zeta potential of the optimized NCS formulation determined using Malvern Zetasizer Nano series Nano-ZS, were found to be $143.5 \mathrm{~nm}, 0.269$ and $-36.6 \mathrm{mV}$ respectively, as shown in fig. 3. Most NCS formulations produced by high-pressure homogenization ( $\mathrm{HPH})$ are characterized by an average particle size below $500 \mathrm{~nm}$, the attainable particle size is especially dependent on the concentration of stabilizer, pressure and numbers of cycles in HPH. Low PDI value $(<0.3)$ indicated the narrow distribution of size (monodispersity) and stability of the formulation was indicated by the zeta potential value $(-36.6 \mathrm{mV})$. Zeta potential is an important physicochemical parameter that influences the stability of nano suspensions.

Extremely positive or negative zeta potential values cause larger repulsive forces, whereas repulsion between particles with similar electric charge prevents aggregation of the particles and thus ensures easy redispersion. A minimum zeta potential of $\pm 20 \mathrm{mV}$ is desirable for the stabilization achieved by steric stabilizers [27].

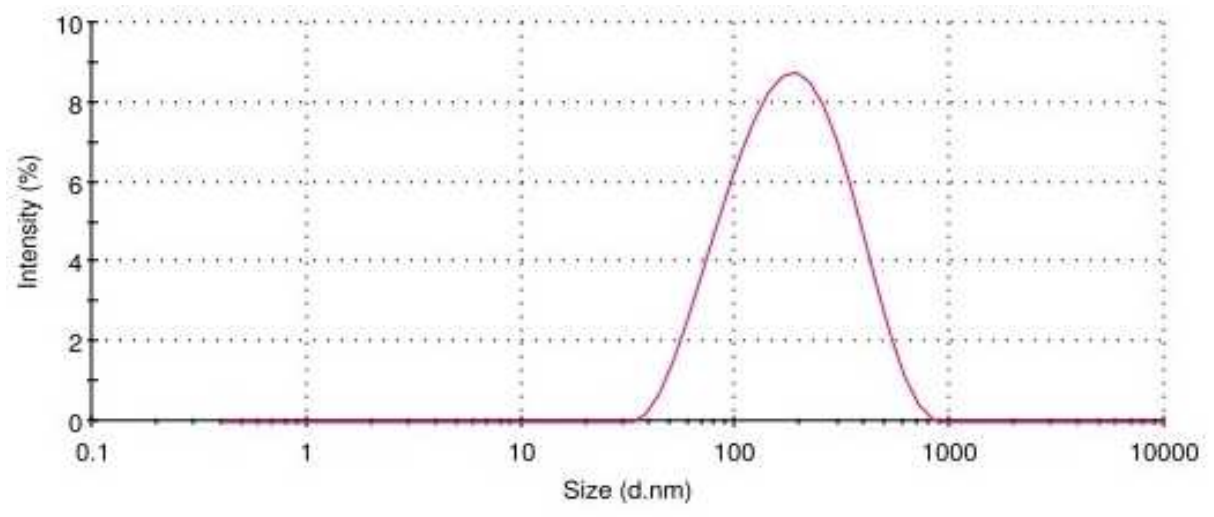

Record 159: cef

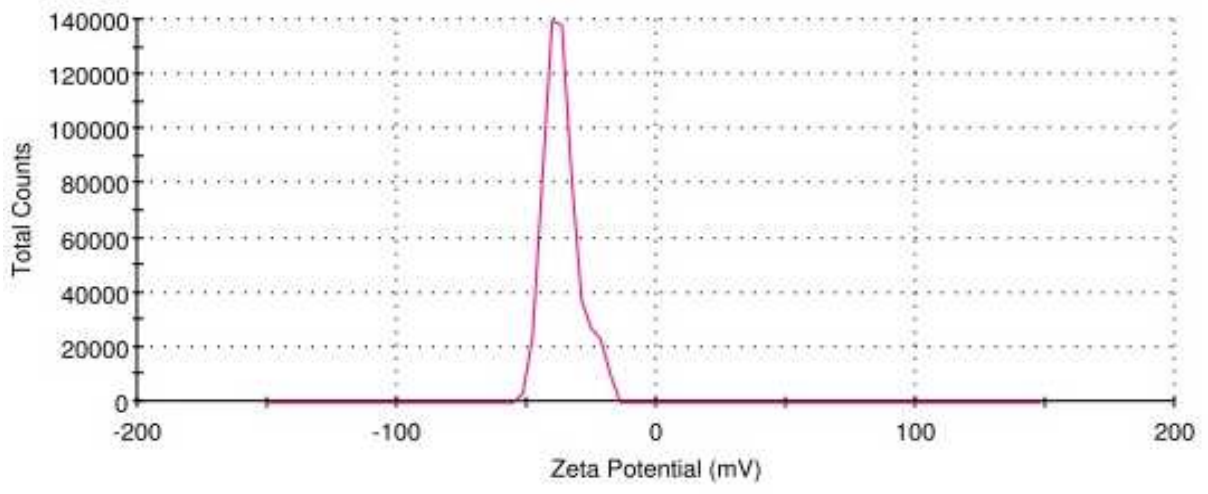

Record 158: cef zeta

Fig. 3: Particle size and zeta potential of optimized nanocrystal suspension formulation

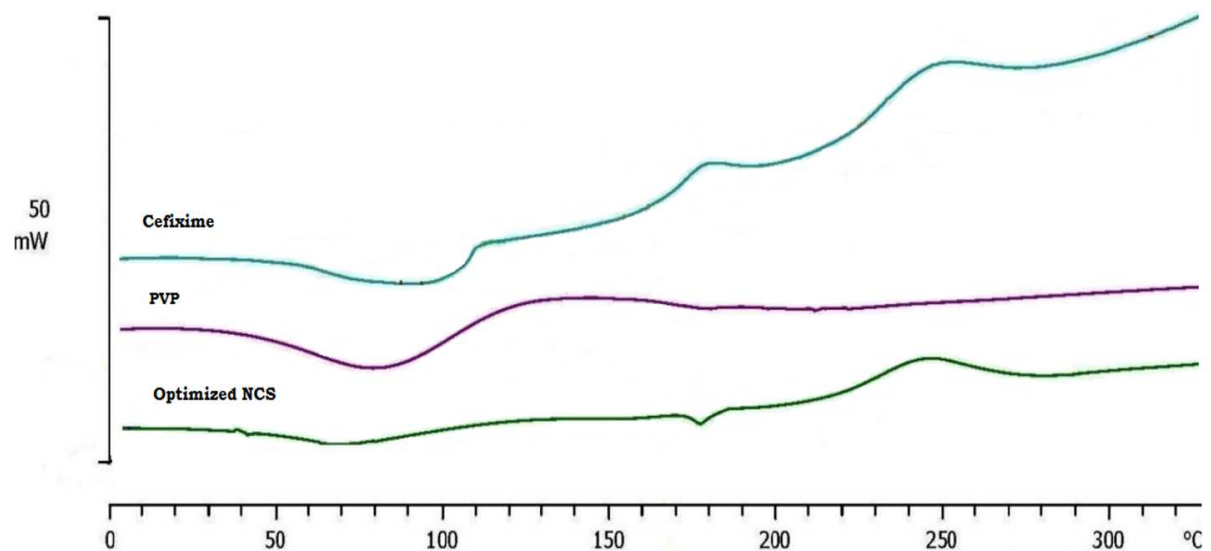

Fig. 4: Differential scanning calorimetry thermogram of pure drug, stabilizer and optimized formulation 


\section{Differential scanning calorimetry (DSC)}

The DSC thermo grams of cefixime trihydrate, PVP K-30 and optimized formulation have been represented in fig. 4, which can provide valuable information about the physiochemical state of nanoparticles.

The influence of the heat flow on the thermal properties of stabilizer, drug, and optimized formulation are illustrated. For raw cefixime trihydrate, the endothermic peak around $100-110^{\circ} \mathrm{C}$ represents the evaporation of water molecules from the crystal lattice and exothermic peaks at $215{ }^{\circ} \mathrm{C}$ and $250{ }^{\circ} \mathrm{C}$ could be related to the crystalline state transition and drug decomposition, respectively. In the thermogram of PVP K-30, a broad endothermic peak ranging from corresponding to the loss of water was observed and can be due to the hygroscopic nature of PVP polymers. In CFX nanoparticles thermogram, both endothermic peaks of CFX and PVP K-30 were shifted to $180^{\circ} \mathrm{C}$ and the exothermic peak of CFX decomposition was still visible at $250^{\circ} \mathrm{C}$, indicating the stability of optimized NCS formulation.

\section{Fourier transform infrared spectroscopy (FTIR) studies}

Fourier transform infrared spectroscopy of cefixime trihydrate, physical mixture and optimized NCS have revealed important information regarding possible interactions between functional groups of added ingredients in the formulation and are presented in fig. 5 .

The changes observed i.e. frequency shifts/disappearance, attenuation and broadening in characteristics peaks of the optimized formulation were observed, which could be attributed to the loss of water from crystal lattice and slight crystalline to amorphous transition. FT-IR results indicate the presence and stability of the drug in the optimized formulation.
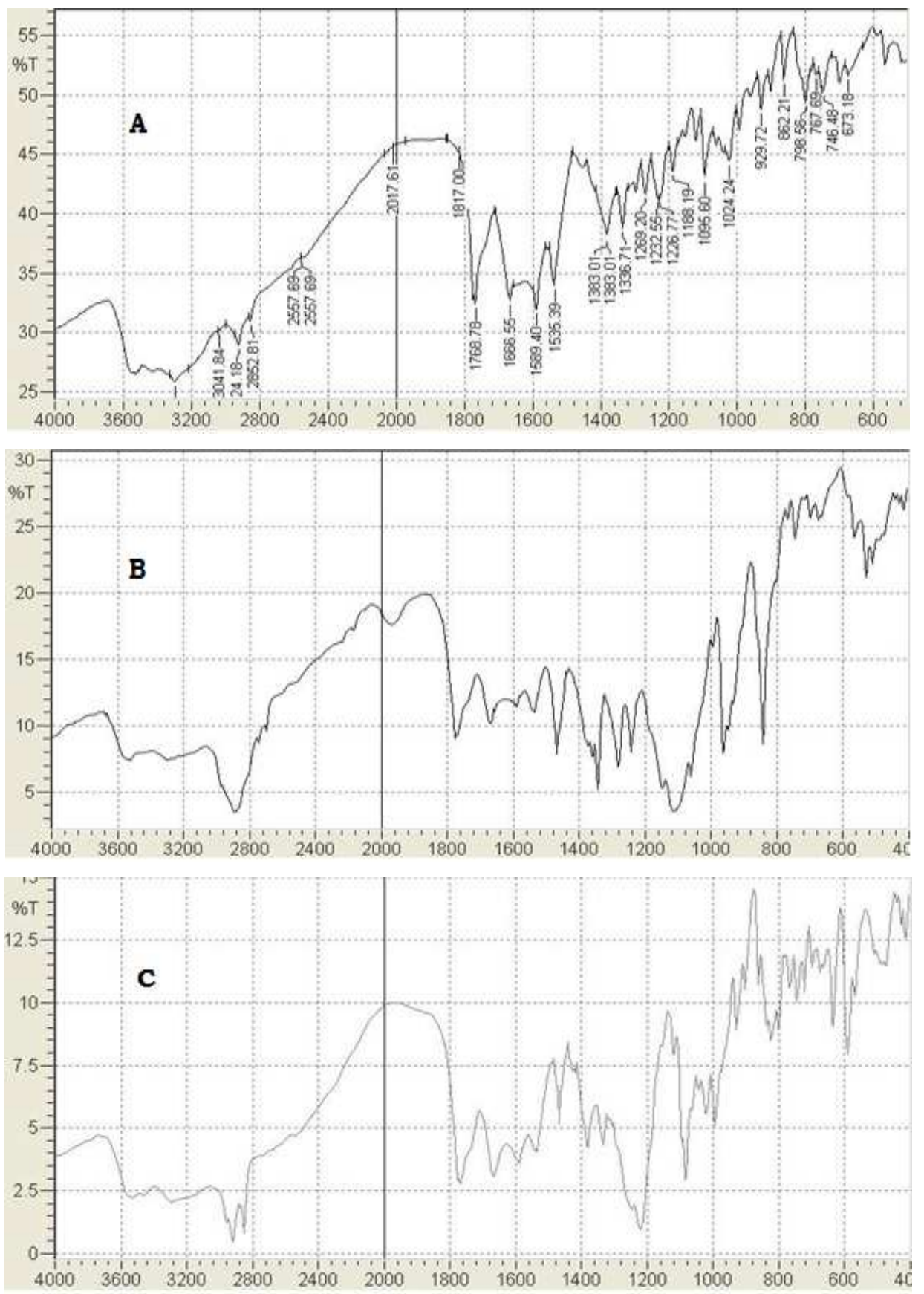

Fig. 5: Fourier transform infra-red spectra of drug (A), physical mixture (B) and optimized formulation (C) 


\section{Scanning electron microscopy (SEM)}

SEM images of the raw drug and optimized NCS (freeze dried) formulations are shown in fig. 6. It can be observed that raw cefixime trihydrate was of crystalline nature with rough surfaces and optimized formulation was relatively spherical shaped. It indicates that stabilizer may have affected the morphology of optimized formulation and thus contribute towards the stability of the optimized formulation. Enhanced morphological properties contribute towards better performance characteristics [20-22].

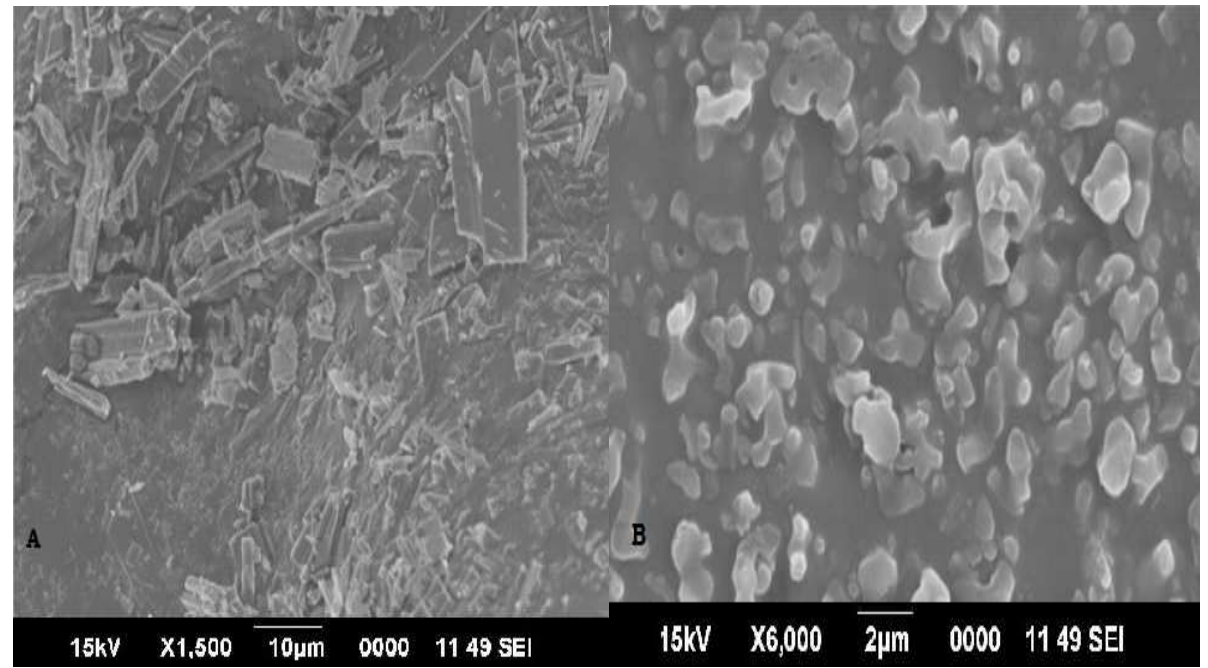

Fig. 6: Scanning electron microscopy images of pure cefixime trihydrate (A) and optimized formulation (B)

\section{Drug release profile}

In-vitro drug diffusion profile of the optimized NCS formulation was obtained by dialysis-bag/dialysis-sac method and was compared with that of plain drug suspension (PDS). The results obtained are as shown in fig. 7. The release of drug from the optimized formulation was found to be more consistent in comparison to the release from a plain drug suspension. $89.79 \pm 0.115 \%$ drug was released from optimized formulation compared to $62.29 \pm 0.173 \%$ drug release from plain drug solution used as a control in $12 \mathrm{~h}$.

The small size of nanoparticles and the presence of stabilizer in the developed optimized formulation may have improved the solubilization, dissolution and resulted in enhanced drug release.

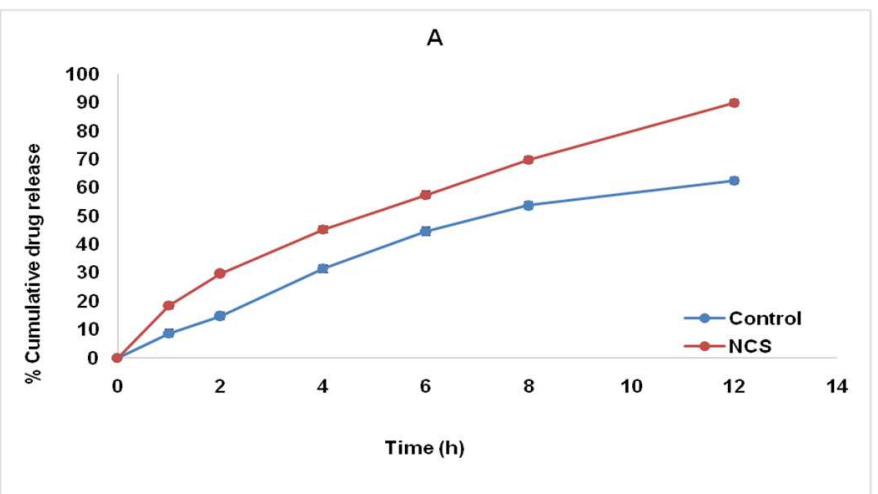

Fig. 7: Drug release profile of optimized formulation vs plain drug solution (control). Error bar indicates mean \pm SD of triplicate experiments

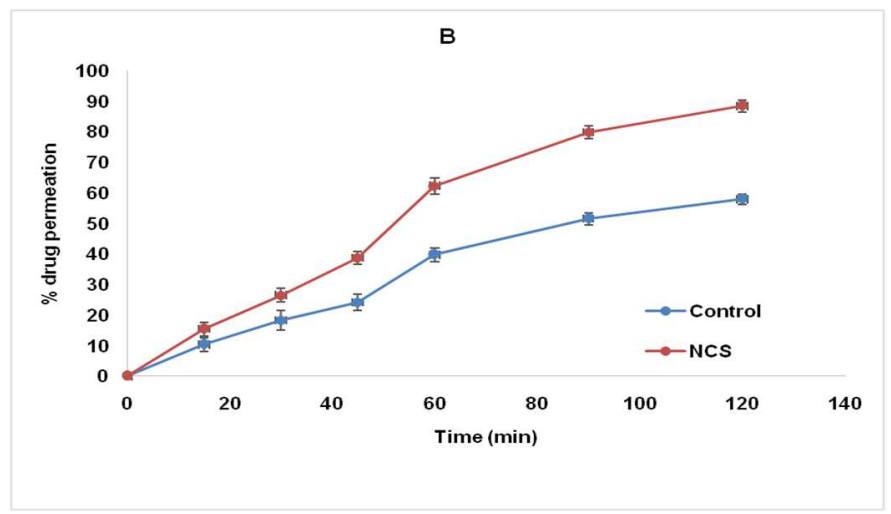

Fig. 8: $E x$-vivo permeation profile of optimized formulation vs plain drug (control). Error bar indicates mean $\pm S D$ of triplicate experiments 


\section{Permeation study}

The permeation study revealed that $88.38 \pm 0.134 \%$ drug within $2 \mathrm{~h}$ was permeated from optimized formulation compared to $57.91 \pm 0.165 \%$ of control (pure drug solution) as showing in fig. 8, indicating increased permeation rate of the drug from the intestinal barrier. This effect is attributed to improved physiochemical properties of optimized formulation compared to free drug.

\section{CONCLUSION}

With the help of results obtained from present investigations, it may be concluded that nanocrystal suspension of a poorly aqueous soluble drug cefixime trihydrate was successfully formulated and can be optimized using the factorial design approach of the design of experiments (DoE) by high-pressure homogenization technique. In present study, cefixime trihydrate NCS was obtained with a mean size of $143.5 \mathrm{~nm}$ (PDI $=0.269$ ) without any additive or organic solvents. Increased saturation solubility can lead to dose reduction with a probability of elimination high dose related side effects. Higher solubility and dissolution rate in optimized NCS formulation can lead to increased drug release and permeation performance reflected by better results in drug release and permeation compared to control formulation (Plain drug solution). Thus, it may be concluded that the developed formulation has better potential for increased solubility and bioavailability.

\section{ACKNOWLEDGMENT}

The authors would like to thank UGC, India, for providing a minor research grant to conduct the present study. The authors are also thankful to R C Patel Institute of Pharmaceutical Education and Research for providing the infrastructural and other facilities for conducting the experiments.

\section{CONTRIBUTION OF AUTHORS}

Both the authors had contributed equally for the presented research work.

\section{CONFLICT OF INTERESTS}

The authors declare that they have no competing interests

\section{REFERENCES}

1. Lipinski CA. Poor aqueous solubility-an industry wide problem in drug discovery. Am Pharm Rev 2002;5:82-5.

2. Maraie NK, T Alhamdany AN, Radhi AA. Efficacy of combination solid dispersion technology on dissolution performance of nalidixic acid and cefdinir. Asian J Pharm Clin Res 2017;10:394-401.

3. Rabinow BE. Nanosuspensions in drug delivery. Nature Rev Drug Discovery 2004;3:785-96.

4. Valeh-e-Sheyda P, Rahimi M, Adibi H, Razmjou Z, Ghasempour H. An insight on reducing the particle size of poorly-water soluble curcumin via LASP in micro channels. Chem Eng Proc: Process Intensification 2015;91:78-88.

5. Han HK, Choi HK. Improved absorption of meloxicam via salt formation with ethanolamines. Eur J Pharm Biopharm 2007;65:99-103.

6. Choi WS, Kim HI, Kwak SS, Chung HY, Chung HY, Yamamoto K, et al. Amorphous ultrafine particle preparation for improvement of bioavailability of insoluble drugs: grinding characteristics of fine grinding mills. Int J Miner Process 2004;74:165-72.

7. Choi JY, Yoo JY, Kwak HS, Nam BU, Lee J. Role of polymeric stabilizers for drug nano crystal dispersions. Curr Appl Phys 2005;5:472-4.

8. Paradkar A, Ambike AA, Jadhav BK, Mahadik K. Characterization of curcumin-PVP solid dispersion obtained by spray drying. Int J Pharm 2004;271:281-6.

9. Gao L, Zhang D, Chen M, Zheng T, Wang S. Preparation and characterization of an oridonin nanosuspension for solubility and dissolution velocity enhancement. Drug Dev Ind Pharm 2007;33:1332-9.
10. Chen Y, Zhang G, Neilly J, Marsh K, Mawhinney D, Sanzgiri Y. Enhancing the bioavailability of ABT-963 using solid dispersion containing Pluronic F-68. Int J Pharm 2004;286:69-80.

11. Jun SW, Kim MS, Kim JS, Park HJ, Lee S, Woo JS, et al. Preparation and characterization of simvastatin/hydroxypropyl- $\beta$-cyclodextrin inclusion complex using supercritical antisolvent (SAS) process. Eur J Pharm Biopharm 2007;66:413-21.

12. Sweetman SC. Martindale: the complete drug reference. $34^{\text {th }}$ ed. Pharmaceutical Press. London and Chicago; 2005. p. 900-1.

13. Paul Y, Kumar M, Singh B. Formulation and in vitro evaluation of Gastroretentive drug delivery system of cefixime trihydrate. Int J Drug Dev Res 2011;3:148-61.

14. Arora S, Sharma P, Irchhaiya R, Khatkar A, Singh N, Gagoria J. Development, characterization and solubility study of solid dispersion of cefpodoxime proxetil by solvent evaporation method. Int J ChemTech Res 2010;2:1156-62.

15. Khan FA, Zahoor M, Islam NU, Hameed R. Synthesis of cefixime and azithromycin nanoparticles: an attempt to enhance their antimicrobial activity and dissolution rate. J Nanomater 2016. http://dx.doi.org/10.1155/2016/6909085

16. Choi SH, Lee JS. Inventors; international health management associates, Inc., assignee. Complexes to improve oral absorption of poorly absorbable antibiotics. United States patent US; 2001. p. 360

17. Wenzel U, Kuntz S, Diestel S, Daniel H. PEPT1-mediated cefixime uptake into human intestinal epithelial cells is increased by $\mathrm{Ca}^{2+}$ channel blockers. Antimicrob Agents Chemother 2002;46:1375-80.

18. Loftsson T, Muellertz A, Siepmann J. For the special IJP issue "Poorly soluble drugs". Int J Pharm 2013;1:1-2.

19. Bevernage J, Brouwers J, Brewster ME, Augustijns P. Evaluation of gastrointestinal drug supersaturation and precipitation: strategies and issues. Int J Pharm 2013;453:25-35.

20. Müller RH, Peters K. Nanosuspensions for the formulation of poorly soluble drugs: I. Preparation by a size-reduction technique. Int J Pharm 1998;160:229-37.

21. Möschwitzer JP. Drug nanocrystals in the commercial pharmaceutical development process. Int J Pharm 2013;453:142-56.

22. Müller RH, Gohla S, Keck CM. State of the art of nanocrystalsspecial features, production, nanotoxicology aspects and intracellular delivery. Eur J Pharm Biopharm 2011;78:1-9.

23. Keck CM, Müller RH. Drug nanocrystals of poorly soluble drugs produced by high-pressure homogenization. Eur J Pharm Biopharm 2006;62:3-16.

24. Van Eerdenbrugh B, Van den Mooter G, Augustijns P. Top-down production of drug nanocrystals: nanosuspension stabilization, miniaturization and transformation into solid products. Int J Pharm 2008;364:64-75.

25. Wang Y, Zheng Y, Zhang L, Wang Q, Zhang D. Stability of nano suspensions in drug delivery. J Controlled Release 2013;172:1126-41.

26. Deng Z, Xu S, Li S. Understanding a relaxation behaviour in a nanoparticle suspension for drug delivery applications. Int J Pharm 2008;351:236-43.

27. Wu L, Zhang J, Watanabe W. Physical and chemical stability of drug nanoparticles. Adv Drug Delivery Rev 2011;63:456-69.

28. Verma S, Huey BD, Burgess DJ. Scanning probe microscopy method for nanosuspension stabilizer selection. Langmuir 2009;25:12481-7.

29. Gassmann P, List M, Schweitzer A, Sucker H. Hydrosols: alternatives for the parenteral application of poorly watersoluble drugs. Eur J Pharm Biopharm 1994;40:64-72.

30. Abdelwahed W, Degobert G, Stainmesse S, Fessi H. Freezedrying of nanoparticles: formulation, process and storage considerations. Adv Drug Delivery Rev 2006;58:1688-713.

31. Chaubal MV, Popescu C. Conversion of nanosuspensions into dry powders by spray drying: a case study. Pharm Res 2008;25:2302-8.

32. Gao Y, Zuo J, Bou-Chacra N, Pinto TD, Clas SD, Walker RB, et al. In vitro release kinetics of anti-tuberculosis drugs from nanoparticles assessed using a modified dissolution apparatus. 
BioMed Res Int 2013. http://dx.doi.org/10.1155/2013/ 136590.

33. Gupta S, Chavhan S, Sawant KK. Self-nano emulsifying drug delivery system for adefovir dipivoxil: design, characterization, in vitro and ex vivo evaluation. Colloids Surf A 2011;392:145-55.

34. Quan P, Shi K, Piao H, Piao H, Liang N, Xia D, et al. A novel surface modified nitrendipine nanocrystals with enhancement of bioavailability and stability. Int J Pharm 2012;430:366-71.

35. Gao L, Zhang D, Chen M. Drug nanocrystals for the formulation of poorly soluble drugs and its application as a potential drug delivery system. J Nanopart Res 2008;10:845-62.

36. Benvegnu $T$, Plusquellec $D$, Lemiègre L. Surfactants from renewable sources: synthesis and applications. Monomers Polym Compos Renewable Resour 2008;3:153-78.
37. Tolman JA, Williams III RO. Advances in the pulmonary delivery of poorly water-soluble drugs: influence of solubilization on pharmacokinetic properties. Drug Dev Ind Pharm 2010;3:1-30.

38. Somasundaram I, Kumar SS. Pramipexole dihydrochloride loaded mpeg-pcl nanosuspension by modified nanoprecipitation: in vitro and in vivo evaluation. Asian J Pharm Clin Res 2016;6:161-7.

\section{How to cite this article}

- $\quad$ Rajveer Bhaskar, Prakash Hiraman Patil. Nanocrystal suspension of cefixime trihydrate preparation by high-pressure homogenization formulation design using $2^{3}$ factorial design. Int J Pharm Pharm Sci 2017;9(9):64-71. 